\title{
Proactive Release of Antimicrobial Essential Oil from a "Smart" Cotton Fabric
}

\author{
Danaja Štular ${ }^{1}$, Matic Šobak ${ }^{2}$, Mohor Mihelčič ${ }^{2}$, Ervin Šest ${ }^{2}$, Ilija German Ilić $^{3}{ }^{\mathbb{D}}$, \\ Ivan Jerman ${ }^{2}$, Barbara Simončič ${ }^{1}$ and Brigita Tomšič ${ }^{1, *}$ \\ 1 Department of Textiles, Graphic Arts and Design, Faculty of Natural Sciences and Engineering, University \\ of Ljubljana, Aškerčeva 12, 1000 Ljubljana, Slovenia; danaja.stular@ntf.uni-lj.si (D.Š.); \\ barbara.simoncic@ntf.uni-lj.si (B.S.) \\ 2 National Institute of Chemistry, Hajdrihova 19, 1000 Ljubljana, Slovenia; matic.sobak@ki.si (M.Š.); \\ mohor.mihelcic@ki.si (M.M.); ervin.sest@ki.si (E.Š.); ivan.jerman@ki.si (I.J.) \\ 3 The Department of Pharmaceutical Technology, Faculty of Pharmacy, University of Ljubljana, Aškerčeva 7, \\ 1000 Ljubljana, Slovenia; ilija.german.ilic@ffa.uni-lj.si \\ * Correspondence: brigita.tomsic@ntf.uni-lj.si; Tel.: +386-1-20-03-233
}

Received: 25 March 2019; Accepted: 7 April 2019; Published: 10 April 2019

check for updates

\begin{abstract}
Two temperature and $\mathrm{pH}$ responsive submicron hydrogels based on poly $(\mathrm{N}-$ methylenebisacrylamide), chitosan and $\beta$-cyclodextrines (PNCS/CD hydrogel) with varying poly( $N$-isopropylacrylamide) to chitosan ratios were synthesized according to a simplified procedure, reflecting improved stimuli responsive properties and excellent bio-barrier properties, granted by incorporated chitosan. Hydrogels were applied to cotton-cellulose fabric as active coatings. Subsequently, antimicrobially active savory essential oil (EO) was embedded into the hydrogels in order to develop temperature- and $\mathrm{pH}$-responsive cotton-cellulose fabric with double antimicrobial activity, i.e., bio-barrier formation of chitosan along with the proactive release of savory EO at predetermined conditions. The influence of the hydrogels chemical composition on stimuli responsive and antibacterial properties were assessed. Both PNCS/CD hydrogels showed stimuli responsiveness along with controlled release of savory EO. The chemical composition of the hydrogels strongly influenced the size of the hydrogel particles, their temperature and $\mathrm{pH}$ responsiveness, and the bio-barrier forming activity. The increased concentration of chitosan resulted in superior overall stimuli responsiveness and excellent synergy between the antimicrobial activities of the hydrogel and released savory EO.
\end{abstract}

Keywords: antimicrobial activity; chitosan; essential oils; smart textiles; stimuli-responsive hydrogel; $\beta$-cyclodextrin

\section{Introduction}

Essential oils (EOs), as one of the important representatives of the natural antimicrobial agents, play a vital role in the textile industry. Namely, they are used to produce biocompatible, environmentally friendly and non-toxic high value-added products with antimicrobial, anticancer, antioxidant and aromatherapeutic properties, intended for the use as cosmetotextiles or medical textiles [1-4]. However, the application of EOs in textiles is limited due to their sensitivity to various environmental conditions. Namely, some volatile compounds are lost if EOs are stored at high temperatures, while other components become unstable due to variations in the $\mathrm{pH}$ or are likely to undergo the oxidation process and generation of free radicals when the EOs are exposed to the combination of oxygen and light [5].

One possible approach for the incorporation of EOs into textile materials is their embedment into stimuli-responsive hydrogels. In this manner, the EOs are not only protected against the 
environment but are also proactively released from the hydrogel matrix only at predetermined conditions. Such controlled release of entrapped substances is based on the ability of the hydrogel particles to reversibly swell or de-swell due to the presence or absence of different external stimuli [6]. However, two important issues should be considered when embedding the EOs as an active substance into a stimuli-responsive hydrogel matrix. The first is the hydrophobic nature of EOs because it is known that water is a driving force of hydrogel responsiveness and the second is the concentration-dependent antibacterial activity of the released EOs, and thus the lack of antimicrobial efficiency when the hydrogel is in its swollen phase. In addition, the hydrogel matrix acts as a reservoir of the EOs, meaning that there is limit amount of EOs that can be embedded, which lead to the eventual depletion of the EOs during the product use. The first issue can be successfully resolved by introducing $\beta$-cyclodextrines ( $\beta-C D$ ) into the hydrogel structure. Specifically, because $\beta-C D$ consist of a hydrophobic cavity and a hydrophilic surface, they can form an inclusion complex with appropriately sized molecules [7,8]. Accordingly, a successful incorporation of $\beta$-CD into poly( $N$-vinylcaprolactam)-based hydrogel was demonstrated by Kettle et al. by using a two-step synthesis procedure that included the formation of $\beta-C D$ acrylates in the first step, followed by a further copolymerization of the hydrogel in the second step $[9,10]$. However, the two-step synthesis method of the incorporation of reactive $\beta-C D$ functionalized by acrylic groups into the hydrogel matrix is not only time-consuming but is also rather complex, thus the preparation of cyclodextrin acrylates proceeds according to the sophisticated Schlenk technique where the utilization of a less environmentally susceptible organic solvent is necessary. Yi and co-workers have recently reported on the effective, ecologically more susceptible incorporation of $\beta$-CD into poly-( $N$-isopropylacrylamide) (poly-NiPAAm) based hydrogel via a one-step method directly during the hydrogel synthesis process in order to achieve temperature responsiveness [11]. In this study it was also shown that the presence of $\beta-C D$ was beneficial for the increased thermosensitivity of the poly-NiPAAm, which was ascribed to the formation of $\beta$-CD-poly-NiPAAm conjugation.

The second issue regarding the concentration-dependent antibacterial activity of the released EOs within the hydrogel matrix, remains a rather unexplored topic. Accordingly, one possible approach to extend the life span of the antimicrobial activity, but preserve the other beneficial properties of the EOs, is to combine EOs with bio-barrier forming agents, resulting in the formation of dual antibacterial activity. Namely, the bio-barrier forming agents do not leach from the fibers, but create a biological obstacle for the microorganisms that comes in direct with the fibers, meaning that their concentration does not change with time. In this manner, antimicrobial activity of the functionalized fibers could be obtained even in conditions when the beneficial effect of the EOs is minimized, i.e., the hydrophilic, swollen phase of the hydrogel particles, or when the concentration of EOs on the surface of the fibers would decrease below the limit of efficiency.

Among the stimuli-responsive hydrogels, the poly-NiPAAm/chitosan based hydrogel (PNCS hydrogel) is particularly important owing to its response to both temperature and $\mathrm{pH}$ in physiological range [12]. Accordingly, PNCS hydrogel swells at temperatures below $32{ }^{\circ} \mathrm{C}$ (i.e., below the lower critical solution temperature (LCST) of poly-NiPAAm) and/or $\mathrm{pH}$ below 6.5 (i.e., below the $\mathrm{pK}_{\mathrm{a}}$ of chitosan), while it undergoes the transition to the de-swollen, collapsed phase when the temperature and/or $\mathrm{pH}$ rise above the aforementioned values. Bearing in mind that $\mathrm{pH}$-responsive chitosan is a well-known biocompatible bio-barrier forming antimicrobial agent [13-15], dual antibacterial activity can be achieved without the use of additional bio-barrier forming agents, but rather by the simple increase of the chitosan within the PNCS hydrogel. The proposed mechanism of the present study, showing dual antimicrobial activity, is schematically presented in Figure 1. Moreover, it can be also predicted that the increase in the chitosan concentration will not compromise dual temperature and $\mathrm{pH}$ responsiveness of functionalized PNCS/CD hydrogel, as incorporation of $\beta$-CD increases the thermosensitivity of poly-NiPAAm. 


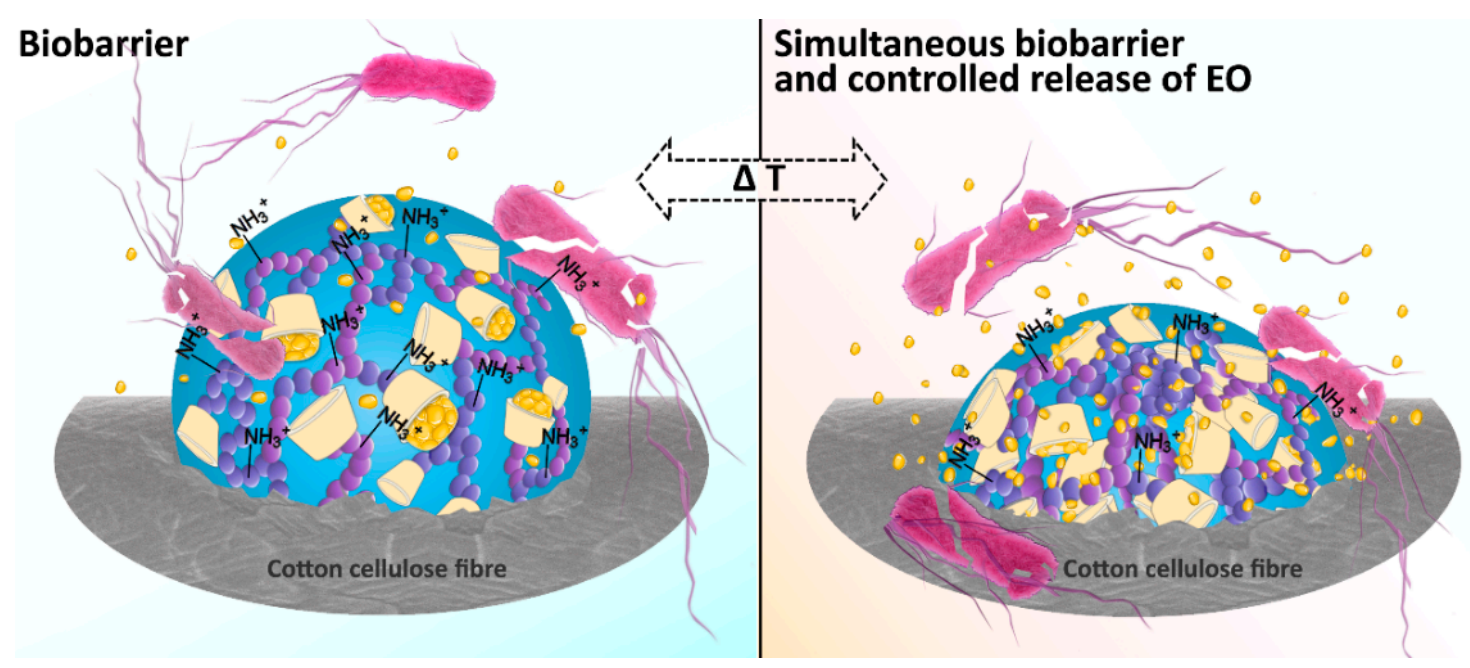

Figure 1. Proposed dual antimicrobial activity of the poly-( $N$-isopropylacrylamide) (poly-NiPAAm)/chitosan based hydrogel (PNCS/CD) hydrogel in correlation with the changes in temperature of the environment.

To the best of our knowledge, there have been no studies about the mutual influence between PNCS/CD hydrogel structure and EOs, thus based on the above consideration, a comprehensive study was performed. Accordingly, this paper focuses on the synthesis PNCS/CD submicron hydrogels with two different poly-NiPAAm to chitosan ratios of 7:1 and 4:1 using a modified in situ synthesis procedure. Subsequently, the PNCS/CD hydrogels were applied to the cotton-cellulose fabric as a thin layer surface modifying system and were functionalized with savory EO, which showed to provide excellent antibacterial activity, without influencing the stimuli responsiveness of the PNCS hydrogel [16]. In this manner, we aimed to study (i) the influence of the $\beta$-CD incorporation on the temperature and/or $\mathrm{pH}$ responsiveness of the PNCS/CD hydrogel, (ii) the influence of the increased chitosan concentration on the temperature responsiveness of the PNCS/CD hydrogel and (iii) the possible achievement of synergistic dual antimicrobial activity derived by the proactive release of savory EO and bio-barrier-forming chitosan.

\section{Materials and Methods}

\subsection{Materials}

In this study, alkaline-scoured, bleached, and mercerized 100\% cotton-cellulose plain weave fabric with a mass area of $125 \mathrm{~g} / \mathrm{m}^{2}$ (warp density: 38 threads $/ \mathrm{cm}$; weft density: 28 threads $/ \mathrm{cm}$ ) was purchased from Tekstina d.d., Ajdovščina, Slovenia. Savory (Satureja montana L.) essential oil (Florihana Distillerie) was purchased from Magnolija, Nina Medved IC, Ljubljana, Slovenia. The chemical composition of savory EO provided by Florihana Destillerie (Caussols, France.) is presented in Supplementary 1 (Table S1). Tween 20 (Sigma-Aldrich, St. Louis, MO, USA) was used as surfactant. For the preparation of the PNCS/CD hydrogel, chitosan (Chitoclear, Primex, Siglufjordur, Iceland; $\mathrm{DD}=95 \% ; \eta=159 \mathrm{mPa}$ ), glacial acetic acid (Sigma-Aldrich), $N$-isopropylacrylamide (NiPAAm) (Sigma-Aldrich), $N, N$-methylenebisacrylamide (MBA) (Sigma-Aldrich), ammonium persulfate (APS) (Sigma-Aldrich), $\beta$-cyclodextrine ( $\beta$-CD) (Sigma-Aldrich) and highly pure sodium dodecyl sulfate (SDS, GE Healthcare Life Sciences, São Paulo, Brazil) were used.

\subsection{Synthesis of the PNCS/CD Hydrogels}

The PNCS/CD hydrogels were synthesized according to the combined and modified procedures firstly reported by Lee [17] and Yi et al. [11]. Briefly, the appropriate concentration of chitosan was dissolved in a mixture of $300 \mathrm{~mL}$ of distilled water and $3 \mathrm{~mL}$ of glacial acetic acid, placed in a flask 
and degassed with nitrogen for $30 \mathrm{~min}$. Next, NiPAAm, $\beta-C D, 10 \mathrm{wt}$ \% SDS solution in water and MBA were added while intensely stirring the mixture at $320 \mathrm{rpm}$ and heating the reaction mixture to $50{ }^{\circ} \mathrm{C}$. After $40 \mathrm{~min}$, APS was added to initiate the polymerization. The mixture turned turbid in approximately $10 \mathrm{~min}$, verifying that the polymerization was proceeding. The reaction proceeded in a nitrogen atmosphere at $50{ }^{\circ} \mathrm{C}$ for $3 \mathrm{~h}$. Subsequently, the PNCS/CD hydrogels reaction mixtures were dialyzed against bidistilled water using a Spectra/Por 4 membrane (Fisher Scientific, Pittsburgh, PA, USA) for 10 days to remove impurities and unreacted monomers and the water was changed daily. The studied PNCS/CD hydrogels have different ratios of poly-NiPAAm and chitosan (PN:CS). The hydrogel sample codes, and the used reagents and their concentrations are presented in Table 1.

Table 1. Synthesis reagents used for the preparation of the PNCS and PNCS/CD hydrogels.

\begin{tabular}{lccccccc}
\hline \multirow{2}{*}{ Hydrogel Code } & \multirow{2}{*}{ PN:CS Ratio } & \multicolumn{7}{c}{ Reagents (g) } \\
\cline { 3 - 7 } & & NiPAAm & Chitosan & $\beta-C D$ & SDS Solution & MBA & APS \\
\hline PNCS/CD M1 & $7: 1$ & 7.00 & 1.00 & 14.00 & 1.50 & 0.21 & 0.90 \\
PNCS/CD M2 & $4: 1$ & 7.00 & 1.75 & 14.00 & 1.55 & 0.22 & 0.93 \\
\hline
\end{tabular}

\subsection{Hydrogel Dispersion Characterization}

The morphology of the hydrogel particles in the dispersions was determined by scanning electron microscopy at an accelerating voltage of $1 \mathrm{kV}$ using an FE-SEM Zeiss SUPRA 35 VP instrument (Oberkochen, Germany). The hydrogel dispersions were freeze-dried, mixed with water, applied on the holder and dried. Prior to the observation, the samples were coated with Pt.

The particle sizes of the dispersed PNCS and PNCS/CD hydrogels as a function of the temperature were determined by dynamic light scattering (DLS) using a Zetasizer NanoS (Malvern, UK) equipped with $4 \mathrm{~mW}$ He-Ne laser operating at the wavelength of $633 \mathrm{~nm}$ and an avalanche photodiode detector. Scattering light was detected at an angle of $173^{\circ}$. Hydrogel was diluted 50 times so that $60 \mu \mathrm{L}$ of the sample was used for each measurement, and the results represent an average of three values. The particle size was determined at $\mathrm{pH} 6.5$ and temperatures in the $20-40{ }^{\circ} \mathrm{C}$ range at intervals of $5{ }^{\circ} \mathrm{C}$.

Thermal analysis was performed by differential scanning calorimetry (DSC) carried out using a DSC 1 calorimeter (Mettler Toledo, Columbus, OH, USA) that was calibrated with indium. Approximately 8-10.5 mg of a hydrogel sample was accurately weighted into a $40 \mu \mathrm{L}$ aluminum pan that was then hermetically sealed with an aluminum lid. The thermal analyses were performed at the temperatures varying from 5 to $50{ }^{\circ} \mathrm{C}$ at a heating rate of $1.5 \mathrm{~K} / \mathrm{min}$ using a nitrogen atmosphere. One cycle of heating and cooling was performed. Normalized thermograms were evaluated using the STARe v9.30 software (Mettler Toledo, Columbus, OH, USA), where the point of the endothermic peak was used to indicate the LCST of the hydrogels.

\subsection{Application of the Hydrogels onto the Cotton-Cellulose Fabric}

PNCS and PNCS/CD hydrogels were applied to a cotton-cellulose fabric using a pad-dry method, which included: immersion of the fabric samples into a hydrogel dispersion, wringing by a two-roller foulard (Mathis, Oberhasli, Switzerland) with a wet pick-up (WP) of $80 \% \pm 5 \%$ and 5 min drying of the samples at $80^{\circ} \mathrm{C}$, using laboratory dryer (Mathis, Oberhasli, Switzerland). The pressure on the foulard varied based on the WP that was calculated for each sample according to [18]:

$$
\mathrm{WP}=\left(\frac{m_{\mathrm{W}}-m_{\mathrm{d}}}{m_{\mathrm{d}}}\right) \times 100[\%]
$$

where $m_{\mathrm{d}}(\mathrm{g})$ is the weight of the dry, untreated sample and $m_{\mathrm{w}}(\mathrm{g})$ represents the weight of the immersed sample after wringing. 


\subsection{Essential Oil Emulsion Preparation}

The appropriate concentration of EO in emulsion was determined according to the disc diffusion test, which provided the information regarding the antimicrobial activity of EO in concentrations from $1 \%$ to 5\%. Results are presented in Supplementary (Figure S1). Based on the results, 5\% savory EO emulsion was prepared for the embedment into the studied PNCS/CD hydrogels. Firstly, $10 \mathrm{~g}$ of Tween 20 was diluted in $85 \mathrm{~g}$ of distilled water and mixed for $20 \mathrm{~min}$ at room temperature with the speed of $100 \mathrm{rpm}$. Then, the stirring speed was set to $1500 \mathrm{rpm}$, while $5 \mathrm{~g}$ of savory EO was added dropwise into the mixture. The mixing proceeded for $4 \mathrm{~h}$ and was followed by ultra-sonication carried out with a lab homogenizer (Hielscher Ultrasonics $\mathrm{GmbH}$, Brandenburg, Germany) for 5 min using 0.5 ultrasonic impulses.

\subsection{Embedment of the EO Emulsion}

Immediately after drying the PNCS/CD coated fabric samples were immersed into a savory EO emulsion, which was previously stored in a refrigerator at $8{ }^{\circ} \mathrm{C}$ in order to fasten the swelling of the shrunken hydrogel particles. The wet samples were pressed with the foulard, to achieve WP of $80 \% \pm 5 \%$. Subsequently, the samples were immersed in a bath containing distilled water:ethanol (60:40) mixture at a goods to liquid ratio of 1:50 in order to remove the excess of the savory EO emulsion.

To characterize the amount of the functional finishing present on the fabric samples, Add-on was determined for each sample. The samples were placed in the moisture analyzer MLB-C (Kern \& SOHN $\mathrm{GmbH}$, Balingen, Germany) and dried to their constant weight. The Add-on was calculated according to following equation [18]:

$$
\text { Add }- \text { on }=\left(\frac{m_{\mathrm{f}}-m_{\mathrm{UN}}}{m_{\mathrm{UN}}}\right) \times 100[\%]
$$

where $m_{\mathrm{f}}(\mathrm{g})$ is the average of the dry weights of the functionalized sample, and $m_{\mathrm{UN}}(\mathrm{g})$ represents an average of dry weights of the untreated sample. Each value represents an average of 10 measurements.

The sample codes and Add-on values of all of the studied samples are presented in Table 2.

Table 2. Sample codes according to the chemical modification of the functionalized cotton-cellulose fabric and corresponding Add-on values.

\begin{tabular}{ccc}
\hline Sample Code & Description of Chemical Modification & Add-on (\%) \\
\hline CO_UN & (a) & $/$ (a) \\
CO_M1 & Cotton fabric treated with PNCS/CD hydrogel M1 (PN:CS $=7: 1)$ & 7.86 \\
CO_M2 & Cotton fabric treated with PNCS/CD hydrogel M2 (PN:CS = 4:1) & 8.89 \\
CO_M1+S & Cotton fabric treated with PNCS/CD hydrogel M1 and savory EO emulsion & 18.19 \\
CO_M2+S & Cotton fabric treated with PNCS/CD hydrogel M2 and savory EO emulsion & 18.88 \\
\hline & (a) No finishing.
\end{tabular}

\subsection{Analysis and Measurements}

\subsubsection{Scanning Electron Microscopy (SEM)}

The morphology of the samples was determined by scanning electron microscopy at an accelerating voltage of $1 \mathrm{kV}$ using an FE-SEM Zeiss SUPRA $35 \mathrm{VP}$ instrument (Oberkochen, Germany). Prior to the observation, the samples were coated with Pt.

\subsubsection{Fourier Transform Infrared (FT-IR) Spectroscopy}

Fourier-transform infrared (FT-IR) spectra were obtained using a Spectrum GX I spectrophotometer (Perkin Elmer, Waltham, MA, USA) equipped with an attenuated total reflection (ATR) cell and a diamond crystal $(n=2.0)$. The spectra were recorded over the range $4000-600 \mathrm{~cm}^{-1}$, with a resolution of $4 \mathrm{~cm}^{-1}$ and averaged over 32 spectra. 


\subsubsection{Moisture Content (MC)}

Temperature responsiveness of the samples was assessed by measuring moisture content according to the AATCC D629-15 Standard Test Methods for Quantitative Analysis of Textiles [19]. For this purpose, a moisture analyzer MLB-C (Kern \& SOHN GmbH, Balingen, Germany) was used. Studied samples were pre-conditioned at $65 \% \pm 2 \%$ relative humidity at 20 and $40{ }^{\circ} \mathrm{C}$ for $24 \mathrm{~h}$, before they were put in a moisture analyzer and dried at $60{ }^{\circ} \mathrm{C}$ until the constant mass. Moisture content (MC) was determined by Equation (3) and each value represents an average of 10 measurements.

$$
\mathrm{MC}=\left(\frac{m_{0}-m_{\mathrm{f}}}{m_{0}}\right) \times 100[\%]
$$

where $m_{0}$ denote the initial mass of the pre-conditioned sample and $m_{\mathrm{f}}$ represents the final mass of the sample after drying. $\mathrm{MC}$ was reported as mean values of 10 measurements.

Based on the results of the average MC value, the contribution $\left(C_{\mathrm{MC}}\right)$ of both hydrogels on the studied samples was calculated by the following equation:

$$
C_{M C}=\left(\frac{\mathrm{MC}_{\mathrm{F}}-\mathrm{MC}_{\mathrm{UN}}}{\mathrm{MC}_{\mathrm{UN}}}\right) \times 100[\%]
$$

where $\mathrm{MC}_{\mathrm{F}}$ is the average moisture content value of the studied functionalized sample, and $\mathrm{MC}_{\mathrm{UN}}$ is the average moisture content value of the untreated sample, determined under the same conditions.

\subsubsection{Water Uptake (WU)}

In order to evaluate $\mathrm{pH}$ responsiveness of the functionalized samples, water uptake was determined [20]. Firstly, the samples were dried to their constant weight using a moisture analyzer MLB-C (Kern \& SOHN GmbH, Balingen, Germany) and their dry weight was determined. Next, they were immersed into the buffer solution with $\mathrm{pH} 3$ for $1 \mathrm{~h}$, followed by immersion for $1 \mathrm{~h}$ in buffer solution with $\mathrm{pH} 8$ and immersion for $1 \mathrm{~h}$ in buffer solution with $\mathrm{pH}$ 3. During the analysis, the samples were taken out of the solutions and weighed every $15 \mathrm{~min}$, thus water uptake (WU) was determined for each measurement according to Equation (5). Each value represents an average of 10 measurements.

$$
\mathrm{WU}=\left(\frac{m_{\mathrm{w}}-m_{0}}{m_{0}}\right) \times 100[\%]
$$

where $m_{\mathrm{w}}$ is the weight of the sample taking up buffer solution $(\mathrm{g})$ and $m_{0}$ is the dry weight of the sample (g).

All data were presented as the mean and standard deviation values. To determine the significance of the differences between studied sets of data, a $t$-test was performed, and the significance limit was set at $p=0.05$.

\subsubsection{Antimicrobial Activity}

Antibacterial activity of studied cotton samples was estimated by determination of zone of inhibition referring to the ISO 20645 Agar diffusion plate test [21], as well as by determination of bacterial reduction according to the ISO 20743 Absorption method [22]. As test organisms, Gram-negative bacteria Escherichia coli (ATCC 25922) and Gram-positive bacteria Staphylococcus aureus (ATCC 6538) were selected.

According to the ISO 20645 Agar diffusion plate test, assessment of antibacterial activity was based on the absence or presence of an inhibition zone, which was calculated from:

$$
H=\frac{D-d}{2}(m m)
$$


where $H$ is the inhibition zone in $\mathrm{mm}, D$ is the total diameter of the cotton specimen and inhibition zone in $\mathrm{mm}$, and $d$ is the diameter of specimen in $\mathrm{mm}$. All tests were performed in duplicate. After the test, the samples and the zone of inhibition were observed with a Leica EZ4 W optical microscope (Leica, Wetzlar, Germany).

According to the ISO 20743 standard method, $1 \mathrm{~g}$ of sample was incubated with bacterial suspension of $10^{5} \mathrm{CFU} / \mathrm{mL}$. The number of surviving colonies was determined by spreading $40 \mu \mathrm{L}$ of suspension on an agar plate immediately after the inoculation (time 0 ) and after $1 \mathrm{~h}$ (time 1 ) of bacteria suspension exposure. The amount of inoculum was adapted to the size of the agar plate. Accordingly, the chosen volume was taken into account in further calculations, adapting to a volume of $1 \mathrm{~mL}$.

The antibacterial activity value was calculated as follows:

$$
A=\left(\lg C_{1}-\lg C_{0}\right)-\left(\lg T_{1}-\lg T_{0}\right)
$$

where $A$ is the antibacterial activity value, $\lg C_{1}$ is the average common logarithm for the number of bacteria obtained from control sample after $1 \mathrm{~h}$ of incubation, $\lg C_{0}$ is the average common logarithm for the number of bacteria obtained from the control sample immediately after inoculation, $\lg T_{1}$ is the average common logarithm for the number of bacteria obtained from tested sample after $1 \mathrm{~h}$ of incubation and $\lg T_{0}$ is the average common logarithm for the number of bacteria obtained from the tested sample immediately after inoculation. For each sample, the values represent the average of eight counts.

\section{Results and Discussion}

\subsection{Characterization of the Hydrogels}

The morphology and particle size of the studied PNCS/CD M1 and M2 hydrogels with different PN:CS ratios were determined by SEM and DLS analysis. In addition, the temperature of the phase change transition of the studied hydrogel particles was also determined. The representative SEM images of these samples are shown in Figure 2. The PNCS/CD M1 hydrogel particles were spherically shaped, but in some cases, the outline of the individual particles was blurred, implying that these particles were coated. In contrast, the spherical shape of the hydrogel particles of PNCS/CD M2 was more distinct with clearly formed individual segments. Since direct comparison between the particle sizes of the two hydrogels was not possible, the DLS method was employed.
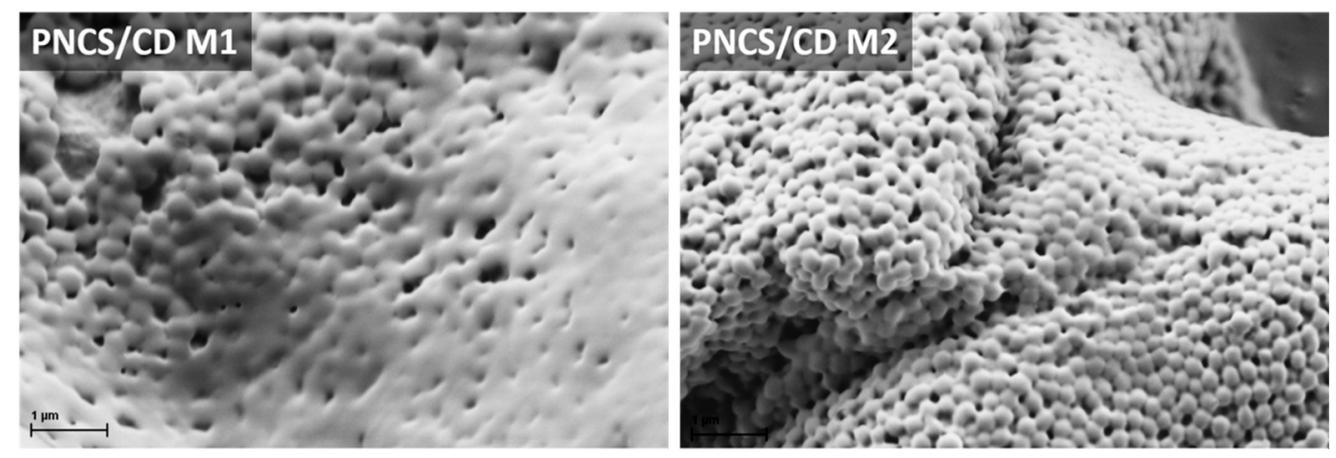

Figure 2. Scanning electron microscope images of the studied PNCS/CD hydrogels.

The results of the hydrodynamic particle size of the studied PNCS/CD hydrogels in correlation with the fluctuation in the temperature of the intermediate surroundings are presented in Figure 3. Regardless of the polymer ratio, both of the studied PNCS/CD hydrogels had submicron size particles at $20^{\circ} \mathrm{C}$, responding to the temperature increase by decreasing their hydrodynamic size. However, for the PNCS/CD M1 hydrogel, this decrease was only obtained until the temperature reached $30{ }^{\circ} \mathrm{C}$, whereas at higher temperatures, the hydrodynamic size of the particles increased 
dramatically. This behavior can be ascribed to the fast agglomeration of the PNCS/CD M1 hydrogel particles at the temperatures above the LCST of poly-NiPAAm, as was confirmed by the drastic increase in the polydispersity index (PDI) values. The rapid agglomeration of the PNCS/CD M1 hydrogel particles can be attributed to the formation of an inclusion complex between the $\beta-C D$ and poly-NiPAAm that enhanced the temperature responsiveness [11]. For the PNCS/CD M2 hydrogel, the agglomeration process was not observed, thus confirming that the presence of higher concentration of chitosan stabilizes the hydrogel particles during the synthesis procedure [23]. Namely, at $20^{\circ} \mathrm{C}$, when poly-NiPAAm is in its hydrophilic state and the particles are swollen, the hydrodynamic particle size of the PNCS/CD M2 hydrogel was 20\% smaller than that obtained for the PNCS/CD M1 hydrogel. The hydrodynamic size was slowly decreased by gradually raising the temperature over the entire studied temperature range, showing the reduction of $66 \%$ in the particle size at the end of the experiment. Furthermore, comparison of the hydrodynamic particle size of the pure PNCS hydrogel that was determined in our previous research [24] to the hydrodynamic particle size of the PNCS/CD M1 hydrogel shows that at the same PN:CS ratio, the inclusion of $\beta$-CD into the hydrogel structure gave rise to an approximately $14 \%$ increase in the particle size. This finding unambiguously demonstrates the beneficial effect of the higher chitosan concentration in the formation of smaller and more stable particles for the PNCS/CD M2 hydrogel.

As observed from Figure 4, the LCST was found at $32.15^{\circ} \mathrm{C}$ for the PNCS/CD M1 hydrogel and at $32.16{ }^{\circ} \mathrm{C}$ for the PNCS/CD M2 hydrogel. Both temperatures are in agreement with the LCST of the linear poly-NiPAAm homopolymer (LCST of approximately $32{ }^{\circ} \mathrm{C}$ ), implying that neither the incorporation of $\beta-C D$ nor the increased chitosan concentration showed any effect on the LCST of the studied hydrogels.

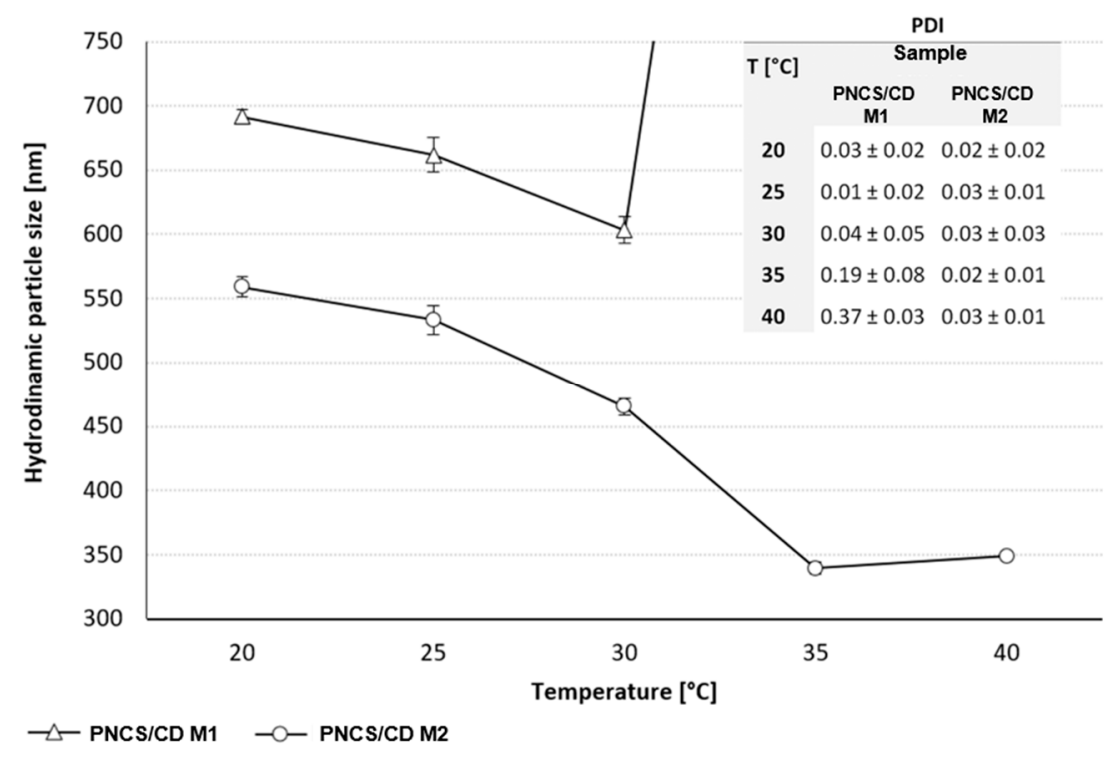

Figure 3. Hydrodynamic PNCS/CD hydrogels particle diameter in the dispersion with the corresponding polydispersity index (PDI) values. 


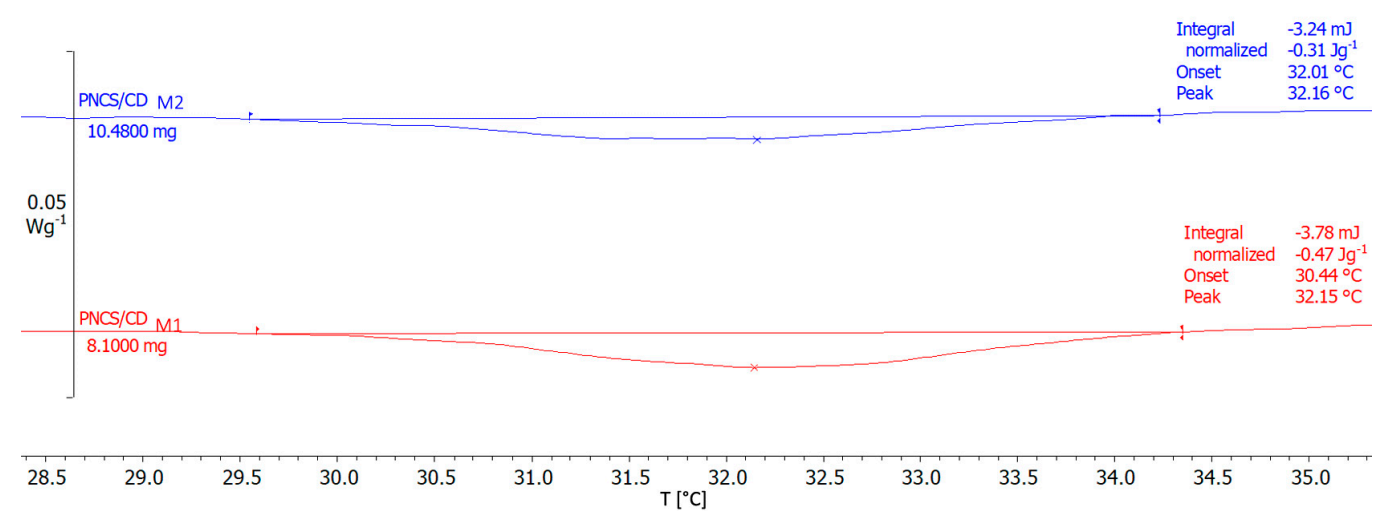

Figure 4. Differential scanning calorimetry (DSC) thermograms of the studied PNCS/CD hydrogels.

\subsection{Morphological and Chemical Properties of the Functionalized Cotton-Cellulose Samples}

The deposition of the stimuli responsive hydrogel altered the morphology of the cotton-cellulose fiber surface due to the presence of spherically shaped bulges (Figure 5). There is a distinct difference in the size of the microgel bulges of both hydrogels applied on cotton fabric. Namely, the hydrogel particles on CO_M1 and CO_M2 samples measured $~ 550$ and $\sim 350 \mathrm{~nm}$ in diameter, respectively. The values were smaller compared to the ones gathered by DLS (Figure 3), due to the vacuum present in SEM machine. Additionally, the water is the driving force of swelling and shrinking of the particles, therefore the particles in dispersion were able to swell freely. Nevertheless, the difference in particle size on the fiber surface correlates to their hydrodynamic size in aqueous media. After in situ embedment of the savory EO emulsion into the PNCS/CD M1 and PNCS/CD M2 hydrogel particles applied on cotton fabric (samples CO_M1+S and CO_M2+S), the volume of the hydrogel bulges visibly increased, which indicates the successful incorporation of EO into the microgel particles.
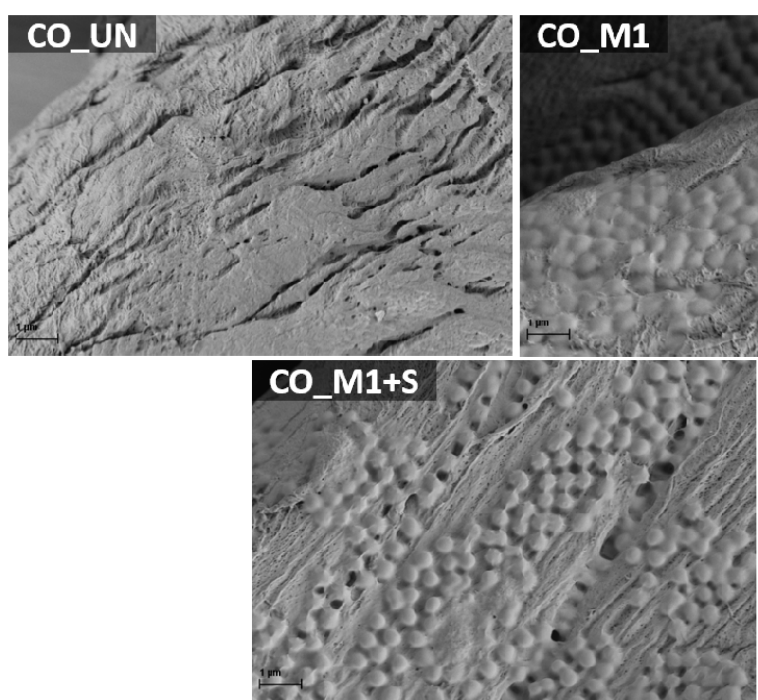
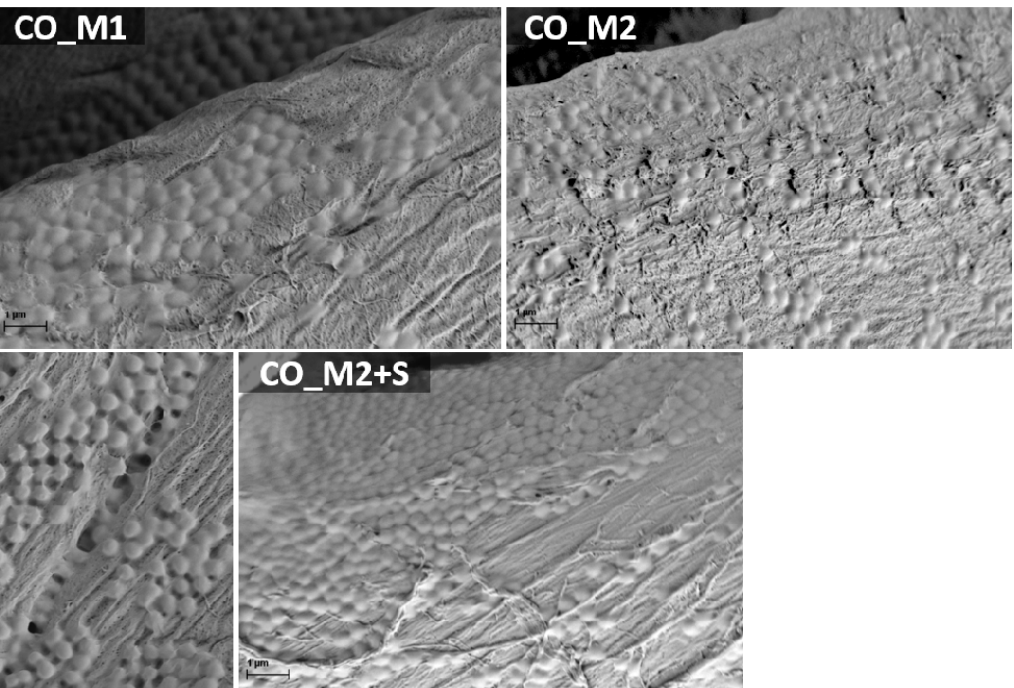

Figure 5. Scanning electron microscopy images of the untreated sample (CO_UN) and the samples finished with bare and functionalized PNCS/CD M1 and M2 hydrogels (CO_M1, CO_M2 and CO_M1+S, CO_M2+S) obtained at the magnification of 25,000 times.

FT-IR ATR spectroscopy was used to obtain information about the characteristic molecular groups and species of the studied surface modification system. To avoid the interference of the characteristic absorption bands of the cotton-cellulose fibers to the characteristic absorption bands of both PNCS/CD hydrogels and the savory EO, the FT-IR ATR analysis was first performed independently of the cotton-cellulose fibers (Figure 6). For this purpose, only the PNCS/CD M1 hydrogel was used, because it was expected that no additional information would be obtained by also analyzing the PNCS/CD 
M2 hydrogel. Accordingly, the PNCS/CD M1 hydrogel was first deposited on a Si wafer, and its IR ATR spectrum was compared to that of the pure PNCS M1 hydrogel in order to confirm the inclusion of $\beta-C D$. An examination of the IR ATR spectrum shows the presence of pure PNCS hydrogel absorption bands belonging to both poly-NiPAAm and chitosan, i.e., the absorption bands at 1630 and $1530 \mathrm{~cm}^{-1}$ that are characteristic of the $\mathrm{C}=\mathrm{O}$ stretching vibration of amide $\mathrm{I}$ and $\mathrm{N}-\mathrm{H}$ deformation vibration of amide II; the absorption band at $3077 \mathrm{~cm}^{-1}$ that is due to N-H stretching; the absorption bands in the $3600-3200 \mathrm{~cm}^{-1}$ spectral region arising due to the $\mathrm{N}-\mathrm{H} \cdots \mathrm{OH}$ and $\mathrm{OH}$ vibrations; the absorption bands at 2970, 2932 and $2875 \mathrm{~cm}^{-1}$ that are ascribed to the stretching vibration of $\mathrm{CH}$ of the $\mathrm{N}$-isopropyl groups and the polymer backbone of poly-NiPAAm and chitosan; the absorption bands in the 1480-1300 cm $\mathrm{cm}^{-1}$ spectral region that are due to the $\mathrm{CH}_{3}$ stretching and $\mathrm{CH}_{3}$ and $\mathrm{CH}_{2}$ deformation vibrations; and, finally, the absorption bands at 1170 and $1130 \mathrm{~cm}^{-1}$ ascribed to the polysaccharide structure of chitosan (Figure 6a) [25-31]. These absorption bands are well observed in the IR ATR spectra of the PNCS/CD M1 hydrogel (Figure 6b), where a conspicuous appearance of the new absorption bands at 1077 and $1035 \mathrm{~cm}^{-1}$ that are ascribed to the $\mathrm{C}-\mathrm{O}-\mathrm{C}, \mathrm{C}-\mathrm{O}$ and $\mathrm{C}-\mathrm{H}$ vibrations indicates the successful introduction of $\beta-C D$ in the PNCS hydrogel [32-34].

Second, the PNCS/CD M1 hydrogel deposited on the Si wafer was in situ functionalized by savory $\mathrm{EO}$ in the same manner as for cotton-cellulose fibers in order to obtain information regarding the EO entrapment into the $\beta$-CD host cavity inside the hydrogel matrix. As expected, in the corresponding spectrum (Figure 6c), the characteristic absorption bands of hydrogel and savory EO (Figure 6d) were observed, with the absorption bands of the PNCS/CD M1 hydrogel dominating. Nevertheless, the presence of the savory EO can be inferred from the strong increase in the absorption band at $3385 \mathrm{~cm}^{-1}$ that is ascribed to a large amount of hydroxyl groups in the water emulsion of savory EO and from the appearance of various absorption bands in the $1730-800 \mathrm{~cm}^{-1}$ spectral region that belong to the various compounds of the savory EO such as carvacrol, thymol, p-cymene and $\gamma$-terpinene that give rise to the absorption bands characteristic of phenols, monoterpene hydrocarbons, alcohols and ketones, sesquiterpenic hydrocarbons and alcohols, aliphatic hydrocarbons, and esters [13,35,36]. In addition, the absorption band of the amide II characteristic of the hydrogel was redshifted from 1530 to $1578 \mathrm{~cm}^{-1}$, indicating a certain interaction between the hydrogel and savory EO emulsion.

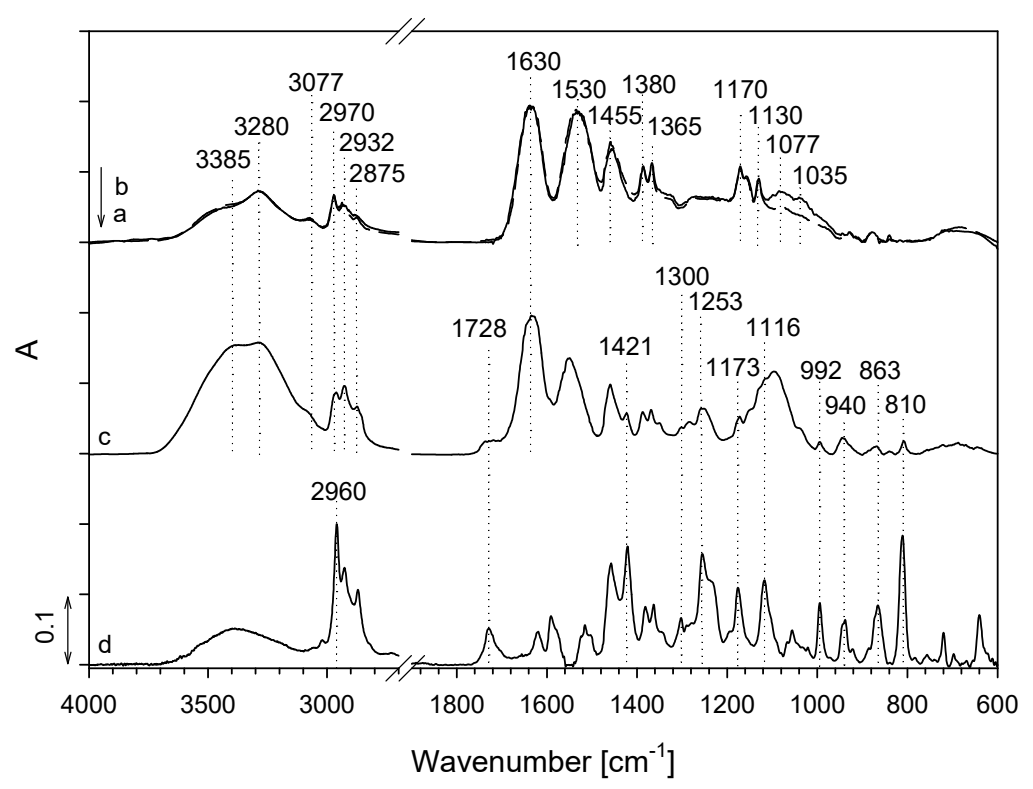

Figure 6. Infrared attenuated total reflection (IR ATR) spectra of the pure PNCS M1 hydrogel (a), PNCS/CD M1 hydrogel before (b) and after in situ functionalization by water emulsion of savory essential oil (EO) (c) and of pure savory EO (d) deposit on a Si wafer. 
A comparison of the ATR IR spectra of the untreated sample with the ATR IR spectra of the functionalized cotton samples CO_M1, CO_M2 and CO_M1+S, CO_M2+S presented in Figure 7 revealed the appearance of absorption bands of amide I and amide II in the ATR IR spectra of both the CO_M1 and CO_M2 samples, thus proving the presence of the PNCS/CD M1 and PNCS/CD M2 hydrogels. The intensities of both absorption bands were slightly higher in the IR ATR spectrum of the CO_M1 sample, indicating the greater concentration of the PNCS/CD M1 hydrogel on the fibers of the CO_M1 sample. After embedding of savory EO, the IR ATR spectra of the CO_M1+S and CO_M2+S samples revealed the presence of the 1728,1465 and $810 \mathrm{~cm}^{-1}$ absorption bands only. The observed absorption bands are in good agreement with those detected in the IR ATR spectrum of pure savory EO, thus clearly proving the presence of savory EO on the functionalized CO_M1+S and CO_M2+S cotton-cellulose samples.

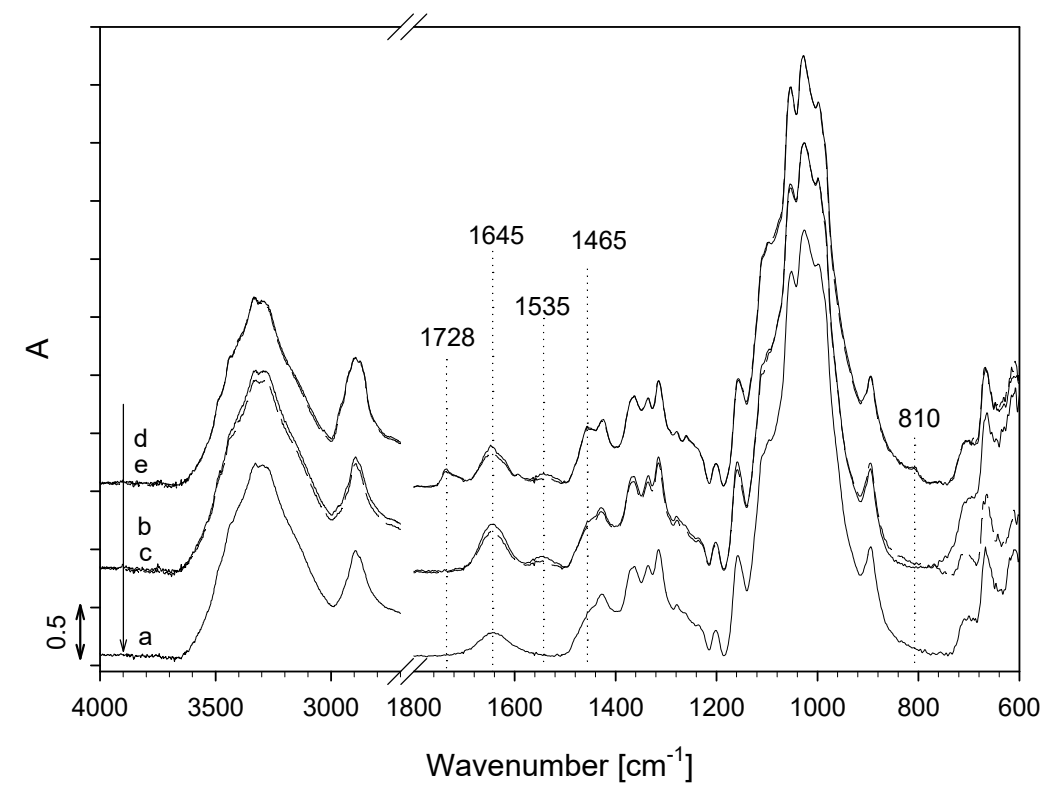

Figure 7. IR ATR spectra of the studied cotton-cellulose samples: CO_UN (a), CO_M1 (b), CO_M2 (c), CO_M1+S (d) and CO_M2+S (e).

\subsection{Responsive Properties}

Responsive properties of the functionalized samples were studied in terms of the temperature and $\mathrm{pH}$ responsiveness. In these studies, only the results for the CO_M1 and CO_M2 samples coated with bare PNCS/CD M1 and PNCS/CD M2 hydrogels are shown and discussed. Namely, due to the presence of volatile compounds, the presence of savory EO strongly interferes with the results obtained for the CO_M1+S and CO_M2+S samples, because a much higher mass difference between the moisturized/wet and completely dry samples was gained. This gave the misleading impression of a higher degree of responsiveness of the CO_M1+S and CO_M2+S samples compared to the CO_M1 and CO_M2 samples.

The results for the temperature responsiveness determined as the moisture content (MC) after the preconditioning of the samples at 20 or $40{ }^{\circ} \mathrm{C}$ and $65 \%$ R.H. are presented in Figure 8a. As expected, at $20^{\circ} \mathrm{C}$, both of the studied hydrogels increased the MC of the CO_M1 and CO_M2 samples compared to the CO_UN sample. This effect was ascribed to their hydrophilic character at the given conditions, resulting in comparable MC values of the functionalized samples that were approximately $13 \%$ higher than that obtained for the CO_UN sample. By contrast, the rise in the temperature triggered the transition of the PNCS/CD M1 and M2 hydrogels from the hydrophilic to the hydrophobic state, resulting in the expulsion of water from their structures. Therefore, at $40^{\circ} \mathrm{C}$, a reduction in the $\mathrm{MC}$ values was observed. In addition, at these conditions, both samples showed comparable MC values 
that were similar to that obtained for the untreated CO_UN sample. Therefore, no useful information could be extracted from the direct comparison of the samples, and thus, the contribution of the surface modifying system to the $\mathrm{MC}\left(\mathrm{C}_{\mathrm{MC}}\right)$ was also determined (Figure $8 \mathrm{~b}$ ). The results demonstrate the superior moisture management of the PNCS/CD M2 hydrogel, which is in agreement with the findings of the DLS analysis (Figure 3), i.e., the beneficial effect of the higher chitosan concentration for the stabilization and reduction in the size of the PNCS/CD M2 hydrogel particles. Specifically, smaller particles have greater specific surface area [6] and therefore exhibit a greater degree of swelling. On the other hand, regarding the influence of the $\beta-C D$ inclusion on the temperature responsiveness of the PNCS hydrogel, it must be mentioned that $\beta-C D$ hindered the moisture management properties compared to the pure PNCS hydrogel, which was well-studied and reported in our previous study [24]. At this point, it is important to note that the impaired moister management of the PNCS/CD hydrogel was reflected in its strong decrease of $\mathrm{MC}$ at $40^{\circ} \mathrm{C}$, showing that the presence of $\beta$-CD strongly affected moisture release. The most likely reason for this is the increased overall polymers' concentration in the PNCS/CD hydrogels due to the inclusion of the $\beta-C D$. Hence, it is known that the release of the moisture from the hydrogel depends on its colloidal properties including high surface area, colloidal stability and control over particle size, as well as their internal network structure characterized by the interaction with embedded compounds, mesh size and polymer volume fraction [6]. In this manner, the higher overall concentration of the polymer networks affects the porosity and tortuosity of the PNCS/CD hydrogels compared to the pure PNCS hydrogel, thus limiting the moisture release at the temperatures inducing the de-swelling of the particles.
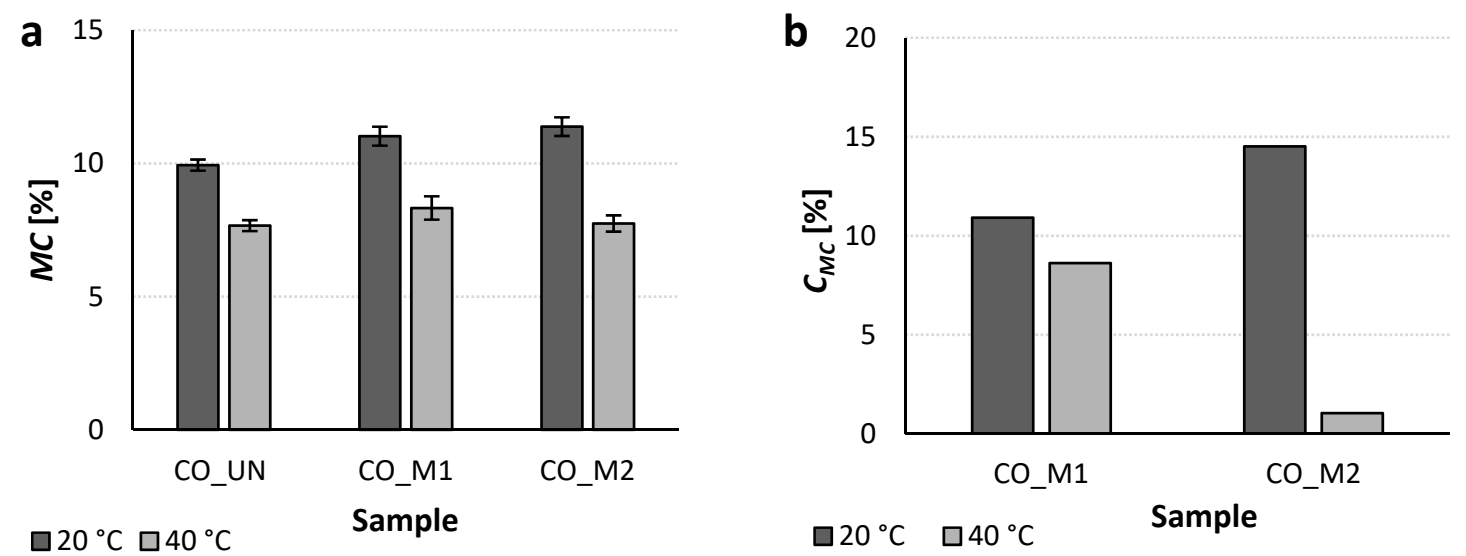

Figure 8. (a) Moisture content (MC) of the studied samples, determined after preconditioning at 20 or $40{ }^{\circ} \mathrm{C}$ and $65 \% \pm 2 \%$ relative humidity for $24 \mathrm{~h}$; (b) contribution of the hydrogels $\left(C_{\mathrm{MC}}\right)$ calculated from the MC values.

To investigate the $\mathrm{pH}$ responsiveness of the functionalized CO_M1 and CO_M2 samples, the water uptake (WU) after their sequential immersion in buffer solutions with $\mathrm{pH} 3$ and 8 was determined. In addition, $p$-values were calculated to ensure that the obtained results were statistically significant (Figure 9).

The CO_UN sample showed no statistically significant difference when transferred from $\mathrm{pH} 3$ to 8 and back, so the greatest increase in WU for this sample occurred within the first 15 min of immersion in $\mathrm{pH} 8$, which can be ascribed to the nature of the cotton-cellulose fibers and their swelling in alkaline conditions [37]. Both the CO_M1 and CO_M2 samples respond to the $\mathrm{pH}$ variations in a similar manner. Namely, the WU dropped when the $\mathrm{pH}$ of the environment rose from 3 to 8 , as the amino groups of chitosan deprotonated and the molecule shrank and expelled the aqueous media. However, when both samples were returned to a buffer solution with acidic $\mathrm{pH}$, the WU increased again, as chitosan was protonated and its molecule extended due to water absorption. As expected, the CO_M2 sample showed the highest WU values due to its highest concentration of the $\mathrm{pH}$-responsive chitosan in the PNCS/CD M2 hydrogel. The differences in WU may seem to be minor, but it should be pointed out 
that the experiment was conducted at room temperature, i.e., when poly-NiPAAm was hydrophilic, so the values only represent the $\mathrm{pH}$ responsiveness caused by chitosan that was present in much lower concentration as the temperature-responsive polymer. Nevertheless, the $p$-values confirm that the differences in the WU values obtained for the CO_M1 and CO_M2 samples were smaller than 0.001 and therefore can be considered to be statistically significant.
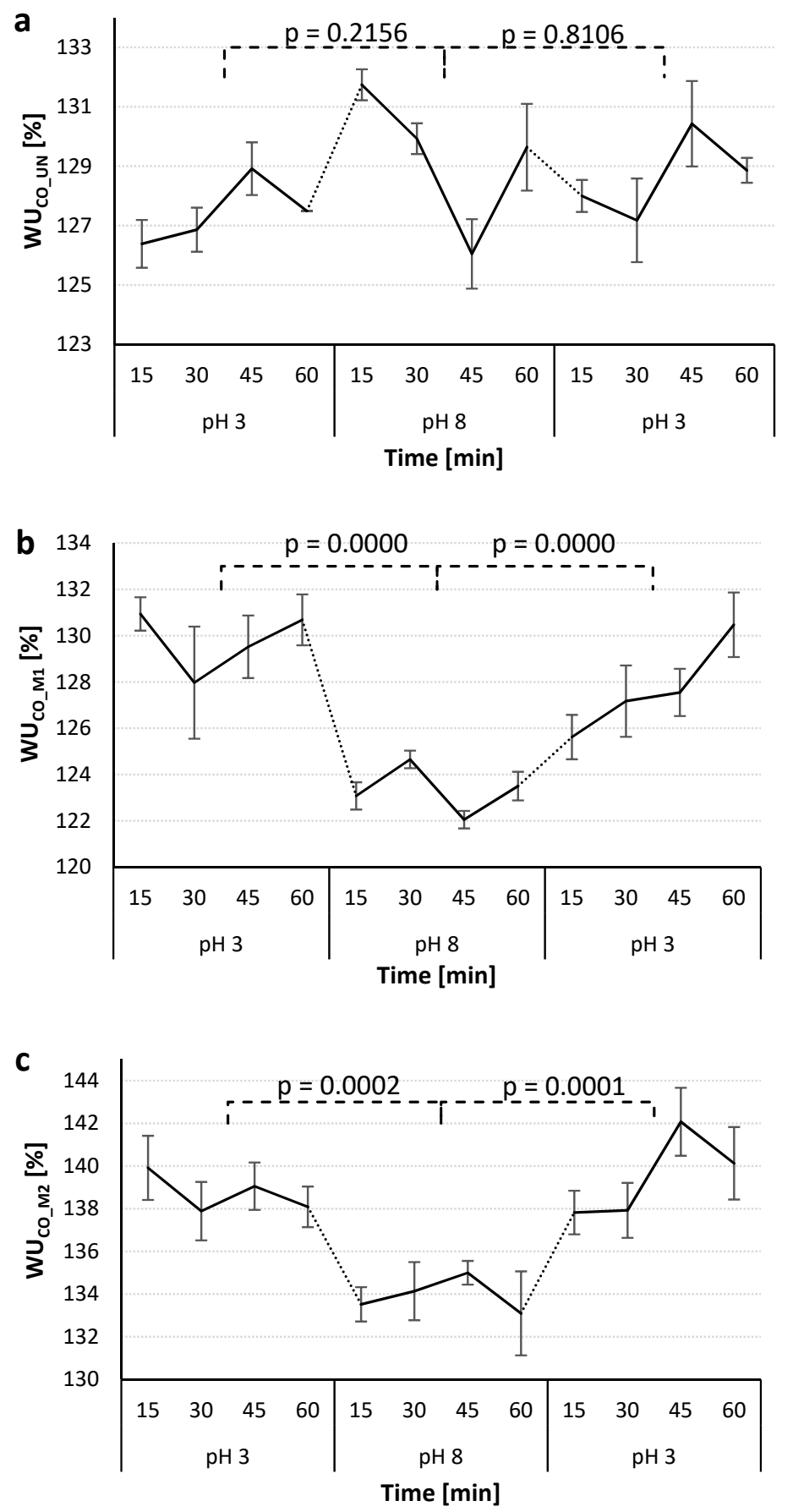

Figure 9. Water uptake (WU) of the studied CO_UN (a), CO_M1 (b) and CO_M2 (c) samples determined each 15 min during alternating $1 \mathrm{~h}$ immersion of the samples in buffer solutions of $\mathrm{pH} 3$ and $\mathrm{pH} 8$.

\subsection{Antimicrobial Activity}

Antibacterial activity was determined in order to verify the controlled release of the savory EO emulsion from the PNCS/CD M1 or PNCS/CD M2 hydrogels deposited on the cotton-cellulose fibers. 
For this purpose, inhibition zone of the gram-negative bacteria E. coli and gram-positive bacteria S. aureus was determined at temperatures below and above the LCST of poly-NiPAAm, i.e., at 23 and $37^{\circ} \mathrm{C}$ (Figure 10). Expectedly, the inhibition zone was not observed for the untreated sample and the CO_M1 and CO_M2 samples, as the bio-barrier formed by the chitosan in the CO_M1 and CO_M2 samples only eliminated the bacteria that came in direct contact with the protonated amino groups of the polymer. By contrast, the CO_M1+S and CO_M2+S samples responded to the temperature changes of the environment, as was indicated by the observed variations in the size of the inhibition zone and the bacteria colonies as the temperature rose from 23 to $37^{\circ} \mathrm{C}$. Namely, when the samples were exposed to temperatures above the LCST of poly-NiPAAm (i.e., $37^{\circ} \mathrm{C}$ ), the hydrogel particles shrunk and squeezed the savory EO out of their structure. At increased concentration, certain compounds of the savory EO invaded the cell membrane, causing either cell lysis or disruption of adenosine triphosphate synthesis, leading to the cell death [38]. Accordingly, an obvious inhibition zone of approximately $1.5 \mathrm{~mm}$ was formed. As seen in Figure 10, there is no distinct difference in the inhibition zone between the CO_M1+S and CO_M2+S samples for both types of bacteria, implying that the increase in the chitosan concentration in the PNCS/CD hydrogels did not affect the proactive release of savory EO.

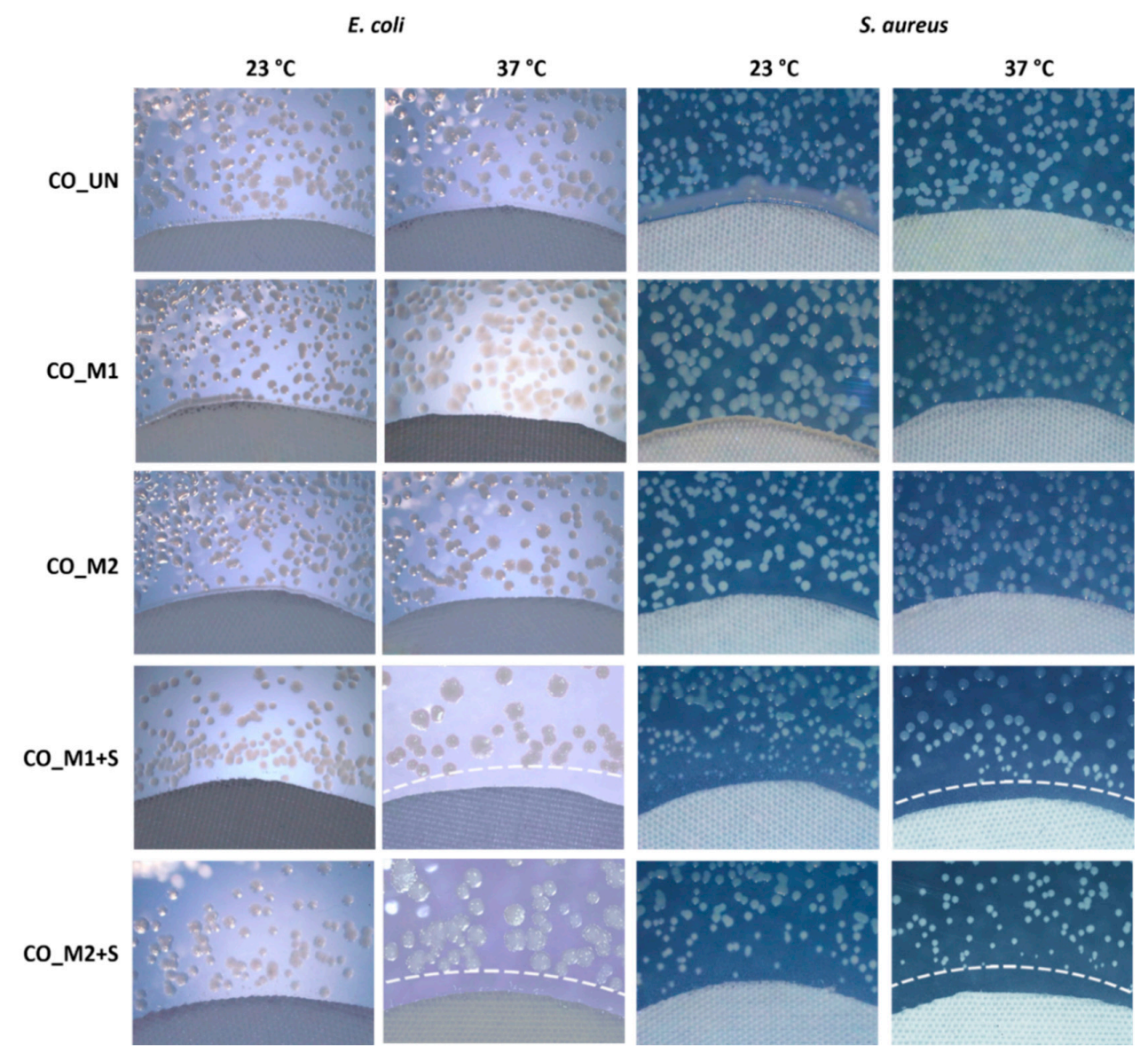

Figure 10. Zone of inhibition formed by the studied cotton-cellulose samples against gram-negative bacteria Escherichia coli and gram-positive bacteria Staphylococcus aureus, after the incubation at 23 or $37^{\circ} \mathrm{C}$.

On the other hand, when the bacteria were allowed to grow at $23^{\circ} \mathrm{C}$, the CO_M1+S and CO_M2+S samples did not form a distinctive inhibition zone, with bacterial colonies growing in the immediate vicinity of the samples. Nevertheless, from a detailed examination of the results it can be observed that in this case the bacterial colonies clearly changed their morphology and size, indicating that their growth was obstructed. This indicates that the slow leaching of savory EO from the hydrogels in the 
swollen state must have occurred. Namely, as proposed by Klinger and Landfester [6], the release of the entrapped active substances from the hydrogel matrix does not proceed only through the squeeze-out mechanism, occurring when the hydrogel particles collapse (de-swell) due to the changes in the environment and expel the entrapped active substances from its structure, but may also occur according to the increased diffusivity mechanism when the hydrogel exists in its swollen phase. In this case, the porosity increases due to the enlarged mesh size of the swollen hydrogel particles and the entrapped substances begin to slowly leak from its structure. Therefore, it can be concluded that savory EO was released from the CO_M1+S and CO_M2+S samples according to both of the mechanisms, but the squeeze-out mechanism was clearly dominant.

In continuation of the research, the antimicrobial activity of the samples against E. coli and S. aureus was analyzed. Based on the number of grown bacteria colonies, the $\log _{10} \mathrm{CFU} / \mathrm{mL}$ was determined (Figure 11). Notably, the higher the $\log _{10} \mathrm{CFU} / \mathrm{mL}$ values, the greater the antibacterial activity of the studied coating against tested bacteria. The results show that the chemical composition of the hydrogels alone had a strong effect on the bacterial reduction, as higher concentration of chitosan in the CO_M2 sample lead to excellent antibacterial activity against both tested bacteria, while the CO_M1 sample showed to be ineffective. In accordance with the results of the zone of inhibition determination, the CO_M1+S and CO_M2+S samples showed increased antibacterial activity against both, E. coli and S. aureus. In this manner, both CO_M1+S and CO_M2+S samples exhibited total bacterial reduction after $1 \mathrm{~h}$ of exposure to the functionalized cotton samples. Comparison of antibacterial activity of these sample shows a strong increase of $\log _{10} \mathrm{CFU} / \mathrm{mL}$ value of the CO_M2+S sample, with antibacterial activity against both tested bacteria greater than $7.0 \mathrm{log}$. These results prove the achievement of the proposed synergistic antimicrobial action between the chitosan in the PNCS/CD M2 hydrogel and embedded savory EO, as double bio-barrier and controlled release antimicrobial activity was successfully obtained.

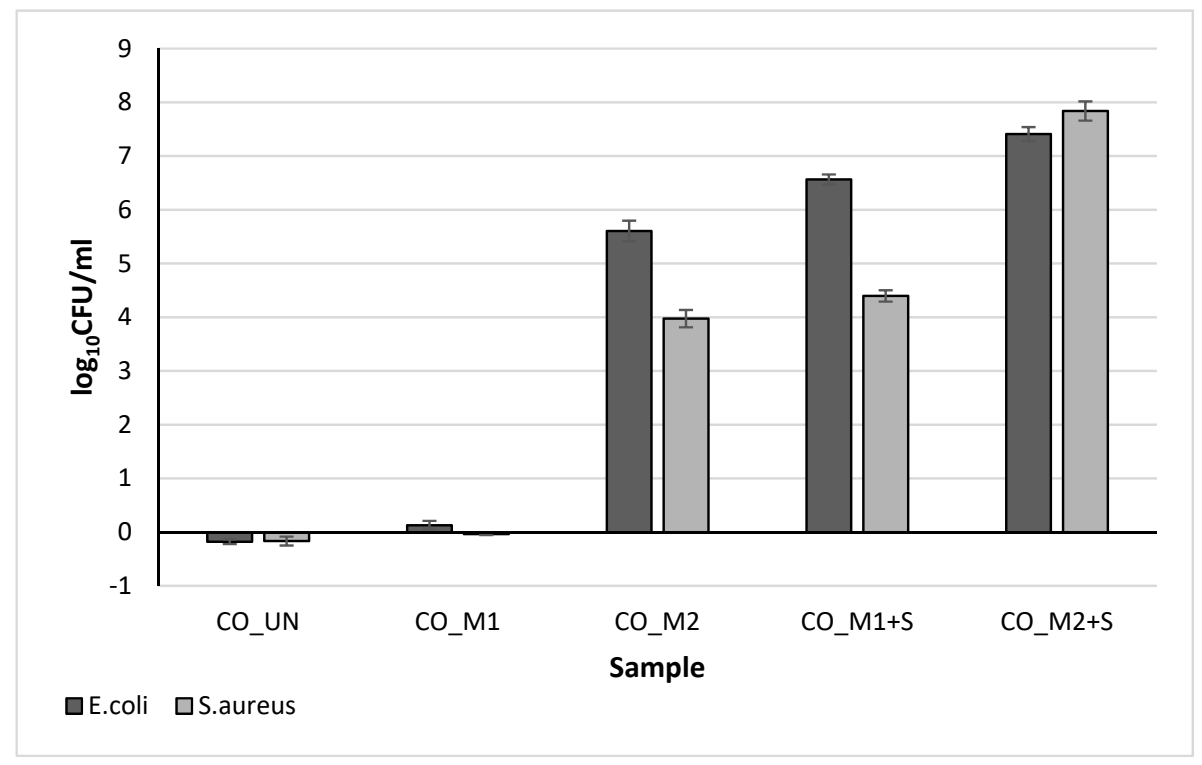

Figure 11. Antimicrobial activity of the studied samples based on the number of grown bacteria colonies right after the exposure to the studied samples and after $1 \mathrm{~h}$ exposure with the samples, determined for E. coli and S. aureus.

\section{Conclusions}

In this work, "smart," temperature and $\mathrm{pH}$ responsive cotton-cellulose fabric with dual antimicrobial activity was tailored. It was shown that chemical composition of the PNCS/CD hydrogels influences the hydrodynamic particle size, with a higher concentration of chitosan reducing the particle sizes and improving the hydrogel particle stability. Accordingly, when present on the surface of the 
cotton-cellulose fibers, PNCS/CD M2 showed superior temperature and $\mathrm{pH}$ responsiveness, the latter derived also due to greater concentration of the protonated amino groups of chitosan. Compared to PNCS hydrogel, the inclusion of $\beta-C D$ in the PNCS/CD hydrogels structure hindered the temperature related release of moisture, due to greater polymer concentration, which decreased the porosity and increased tortuosity of hydrogel particles. Nevertheless, the presence of $\beta-C D$ in the PNCS/CD M1 and PNCS/CD M2 hydrogels enabled the embedment of savory EO in its host cavity that was then released from its structure in a controlled manner, i.e., at elevated temperatures. Successful release of savory EO improved the antibacterial activity of the cotton-cellulose samples as a result of the synergism between bio-barrier forming chitosan within the hydrogels and the embedded savory EO. Accordingly, PNCS/CD hydrogel functionalized cotton fabric could easily be combined with various EOs, thus providing pro-active moisture management and antimicrobial activity along with other beneficial effects of EOs, customized according to the market demand. Such high-added value materials are of great importance in the field of medical textiles and related healthcare and hygiene textiles.

Supplementary Materials: The following are available online at http://www.mdpi.com/2079-6412/9/4/242/s1, Table S1: Essential oil chromatography sheet records provided by the producer of the used EO (Florihana Distillerie, Caussols, France), Figure S1: Zone of inhibition of disc impregnated with (a)-DMSO, and discs impregnated with (b) $-1 \%$, (c) $-2 \%$, (d) $-3 \%$, (e) $-4 \%$ and (f) $-5 \%$ concentration of savory EO in DMSO.

Author Contributions: Investigation, D.Š.; Analysis, D.Š., M.Š., M.M., E.Š., I.G.I.; Writing-Original Draft Preparation, D.Š.; Writing—Review and Editing, B.T., B.S. and I.J.; Supervision, B.T.

Funding: This research was funded by the SLOVENIAN RESEARCH AGENCY (Programme P2-0213, Infrastructural Centre RIC UL-NTF and a grant for the doctoral student D.S..).

Conflicts of Interest: The authors declare no conflict of interest.

\section{Abbreviations}

APS

ATR

$\mathrm{CFU}$

$\mathrm{C}_{\mathrm{MC}}$

CO_M1

CO_M1+S

CO_M2

CO_M2+S

CO_UN

DLS

DSC

E. coli

EO

FT-IR

LCST

MBA

MC

NiPAAm

PDI

$\mathrm{pK}_{\mathrm{a}}$

PN:CS

PNCS hydrogel

PNCS/CD hydrogel

PNCS/CD M1
Ammonium persulfate

Attenuated total reflection

Colony-forming units

Contribution

Cotton fabric treated with PNCS/CD hydrogel M1 (PN:CS = 7:1)

Cotton fabric treated with PNCS/CD hydrogel M1 and savory EO emulsion

Cotton fabric treated with PNCS/CD hydrogel M2 (PN:CS = 4:1)

Cotton fabric treated with PNCS/CD hydrogel M2 and savory EO emulsion

Untreated cotton fabric

Dynamic light scattering

Differential scanning calorimetry

Escherichia coli

Essential oil

Fourier-transform infrared spectroscopy

Lower critical solution temperature

$\mathrm{N}, \mathrm{N}$-methylenebisacrylamide

Moisture content

$\mathrm{N}$-isopropylacrylamide

Polydispersity index

Acid dissociation constant

Poly-NiPAAm to chitosan ratio

Poly-( $N$-isopropylacrylamide)/chitosan based hydrogel

Poly-( $N$-isopropylacrylamide)/chitosan / $\beta$-cyclodextrine based hydrogel

Poly-( $N$-isopropylacrylamide)/chitosan $/ \beta$-cyclodextrine based hydrogel with poly-( $N$-isopropylacrylamide) to chitosan ratio 7:1 


\section{PNCS/CD M2}

poly-NiPAAm

rpm

S. aureus

SDS

SEM

WP

WU

$\beta-C D$
Poly-(N-isopropylacrylamide)/chitosan/ $\beta$-cyclodextrine based hydrogel with poly-( $N$-isopropylacrylamide) to chitosan ratio $4: 1$

Poly-(N-isopropylacrylamide)

Round per minute

Staphylococcus aureus

Sodium dodecyl sulfate

Scanning electron microscopy

Wet pick-up

Water uptake

$\beta$-cyclodextrine

\section{References}

1. Guerra-Rosas, M.I.; Morales-Castro, J.; Cubero-Marquez, M.A.; Salvia-Trujillo, L.; Martín-Belloso, O. Antimicrobial activity of nanoemulsions containing essential oils and high methoxyl pectin during long-term storage. Food Control 2017, 77, 131-138. [CrossRef]

2. Morais, D.; Guedes, R.; Lopes, M. Antimicrobial approaches for textiles: From research to market. Materials 2016, 9, 498. [CrossRef]

3. Islam, S.; Shahid, M.; Mohammad, F. Perspectives for natural product based agents derived from industrial plants in textile applications-A review. J. Clean. Prod. 2013, 57, 2-18. [CrossRef]

4. Solorzano-Santos, F.; Miranda-Novales, M.G. Essential oils from aromatic herbs as antimicrobial agents. Curr. Opin. Biotechnol. 2012, 23, 136-141. [CrossRef] [PubMed]

5. Dima, C.; Dima, S. Essential oils in foods: Extraction, stabilization, and toxicity. Curr. Opin. Food Sci. 2015, 5, 29-35. [CrossRef]

6. Klinger, D.; Landfester, K. Stimuli-responsive microgels for the loading and release of functional compounds: Fundamental concepts and application. Polymer 2012, 53, 5209-5231. [CrossRef]

7. Radu, C.D.; Parteni, O.; Ochiuz, L. Applications of cyclodextrins in medical textiles-Review. J. Control. Release 2016, 224, 146-157. [CrossRef] [PubMed]

8. Zhang, Y.; Zhang, H.; Wang, F.; Wang, L.X. Preparation and properties of ginger essential oil $\beta$-cyclodextrin/chitosan inclusion complexes. Coatings 2019, 8, 305. [CrossRef]

9. Bashari, A.; Hemmatinejad, N.; Pourjavadi, A. Smart and fragrant garment via surface modification of cotton fabric with cinnamon oil/stimuli responsive pnipaam/chitosan nano hydrogels. IEEE Trans. Nanobiosci. 2017, 16, 455-462. [CrossRef] [PubMed]

10. Kettle, M.J.; Dierkes, F.; Schaefer, K.; Moeller, M.; Pich, A. Aqueous nanogels modified with cyclodextrin. Polymer 2011, 52, 1917-1924. [CrossRef]

11. Yi, P.; Wang, Y.; Zhang, S.; Zhan, Y.; Zhang, Y.; Sun, Z.; Li, Y.; He, P. Stimulative nanogels with enhanced thermosensitivity for therapeutic delivery via $\beta$-cyclodextrin-induced formation of inclusion complexes. Carbohydr. Polym. 2017, 166, 219-227. [CrossRef]

12. Jocić, D. Polymer-based smart coatings for comfort in clothing. Tekstilec 2016, 59, 107-114. [CrossRef]

13. Feyzioglu, G.C.; Tornuk, F. Development of chitosan nanoparticles loaded with summer savory (Satureja hortensis L.) essential oil for antimicrobial and antioxidant delivery applications. LWT Food Sci. Technol. 2016, 70, 104-110. [CrossRef]

14. Raafat, D.; Von Bargen, K.; Haas, A.; Sahl, H.G. Insights into the mode of action of chitosan as an antibacterial compound. J. Appl. Environ. Microbiol. 2008, 74, 3764-3773. [CrossRef] [PubMed]

15. Tang, R.; Yu, Z.; Zhang, Y.; Qi, C. Synthesis, characterization, and properties of antibacterial dye based on chitosan. Cellulose 2016, 23, 1741-1749. [CrossRef]

16. Lee, C.F.; Wen, C.J.; Chiu, W.Y. Synthesis of poly(chitosan-nisopropylacrylamide) complex particles with the method of soapless dispersion polymerization. J. Polym. Sci. Part A Polym. Chem. 2003, 41, 2053-2063. [CrossRef]

17. Štular, D.; Jerman, I.; Mihelčič, M.; Simončič, B.; Tomšič, B. Antimicrobial activity of essential oils and their controlled release from the smart PLA fabric. IOP Conf. Ser. Mater. Sci. Eng. 2018, 460, 012011. [CrossRef]

18. Schindler, W.D.; Hauser, P.J. Chemical Finishing of Textiles; Woodhead: Cambridge, UK, 2004. 
19. ASTM D629-15 Standard Test Methods for Quantitative Analysis of Textiles; ASTM International: West Conshohocken, PA, USA, 2015.

20. Kulkarni, A.; Tourrette, A.; Warmoeskerkena, M.M.C.G.; Jocic, D. Microgel-based surface modifying system for stimuli-responsive functional finishing of cotton. Carbohydr. Polym. 2010, 82, 1306-1314. [CrossRef]

21. ISO 20645 Textile Fabrics Determination of Antibacterial Activity Agar Diffusion Plate Test; International Organization of Standards: Geneva, Switzerland, 2004.

22. EN ISO 20743 Textiles-Determination of Antibacterial Activity of Textile Products; European Committee for Standardization: Brussels, Belgium, 2013.

23. Bashari, A.; Hemmatinejad, N.; Pourjavadi, A. Surface modification of cotton fabric with dual-responsive PNIPAAm/chitosan nano hydrogel. Polym. Adv. Technol. 2013, 24, 797-806. [CrossRef]

24. Štular, D.; Jerman, I.; Simončič, B.; Grgić, K.; Tomšič, B. Influence of the structure of a bio-barrier forming agent on the stimuli-response and antimicrobial activity of a "smart" non-cytotoxic cotton fabric. Cellulose 2018, 25, 6231-6245. [CrossRef]

25. Carrillo, F.; Defays, B.; Colom, X. Surface modification of lyocell fibres by graft copolymerisation of thermo-sensitive poly-N-isopropylacrylamide. Eur. Polym. J. 2008, 44, 4020-4028. [CrossRef]

26. Draczyński, Z.; Flinčec Grgac, S.; Dekanić, T.; Tarbuk, A.; Boguń, M. Implementation of chitosan into cotton fabric. Tekstilec 2017, 60, 296-301. [CrossRef]

27. Socrates, G. Infrared and Raman Characteristic Group Frequencies; John Wiley \& Sons: New York, NY, USA, 2001.

28. Gupta, D.; Haile, A. Multifunctional properties of cotton fabric treated with chitosan and carboxymethyl chitosan. Carbohydr. Polym. 2007, 69, 164-171. [CrossRef]

29. Lee, S.B.; Ha, D.I.; Cho, S.K.; Kim, S.J.; Lee, Y.M. Temperature/pH-sensitive comb-type graft hydrogels composed of chitosan and poly(N-isopropylacrylamide). J. Appl. Polym. Sci. 2004, 92, 2612-2620. [CrossRef]

30. Pan, Y.V.; Wesley, R.A.; Luginbuhl, R.; Denton, D.D.; Ratner, B.D. Plasma polymerized $\mathrm{N}$-isopropylacrylamide: Synthesis and characterization of a smart thermally responsive coating. Biomacromolecules 2001, 2, 32-36. [CrossRef]

31. Sun, G.; Zhang, X.Z.; Chu, C.C. Formulation and characterisation of chitosan based hydrogel having both temperature and $\mathrm{pH}$ sensitivity. J. Mater. Sci. Mater. Med. 2007, 18, 1563-1577. [CrossRef]

32. Crupi, V.; Ficarra, R.; Guardo, M.; Majolino, D.; Stancanelli, R.; Venuti, V. UV-vis and FTIR-ATR spectroscopic techniques to study the inclusion complexes of genistein with $\beta$-cyclodextrins. J. Pharm. Biomed. Anal. 2007, 44, 110-117. [CrossRef] [PubMed]

33. Rachmawati, H.; Edityaningrum, C.A.; Mauludin, R. Molecular inclusion complex of curcumin- $\beta$-cyclodextrin nanoparticle to enhance curcumin skin permeability from hydrophilic matrix gel. Aaps Pharm. 2013, 4, 1303-1312. [CrossRef] [PubMed]

34. Wang, H.D.; Chu, L.Y.; Yu, X.Q.; Xie, R.; Yang, M.; Xu, D.; Zhang, J.; Hu, L. Thermosensitive affinity behavior of poly(N-isopropylacrylamide) hydrogels with $\beta$-cyclodextrin moieties. Ind. Eng. Chem. Res. 2007, 46, 1511-1518. [CrossRef]

35. Salmeri, S.; Lacroix, M. Physicochemical properties of alginate/polycaprolactone-based films containing essential oils. J. Agric. Food Chem. 2006, 54, 10205-10214. [CrossRef]

36. Turki, A.; El Oudiani, A.; Msahli, S.; Sakli, F. Infrared spectra for alfa fibers treated with thymol. J. Glycobiol. 2018, 7, 2. [CrossRef]

37. Klemm, D.; Heublein, B.; Fink, H.P.; Bohn, A. Cellulose: Fascinating biopolymer and sustainable raw material. Angew. Chem. Int. Ed. 2005, 44, 3358-3393. [CrossRef]

38. Rieger, K.A.; Schiffman, J.D. Electrospinning an essential oil: Cinnamaldehyde enhances the antimicrobial efficacy of chitosan/poly(ethylene oxide) nanofibers. Carbohydr. Polym. 2014, 113, 561-568. [PubMed]

(C) 2019 by the authors. Licensee MDPI, Basel, Switzerland. This article is an open access article distributed under the terms and conditions of the Creative Commons Attribution (CC BY) license (http:/ / creativecommons.org/licenses/by/4.0/). 